\title{
Temporal integration in global stereopsis
}

\author{
KEITH D. WHITE and J. VERNON ODOM \\ University of Florida, Gainesville, Florida
}

\begin{abstract}
Thresholds for anaglyphic dynamic random element stereograms that reversed in depth were determined using a method of adjustment. Hypercyclopean pattern type (horizontal or vertical gratings, or checkerboards in depth), spatial frequency (0.13-0.55 cpd), and interval between depth reversals $(0.03$ to $4.27 \mathrm{sec})$ were varied. Stereothresholds were lowest at $0.18 \mathrm{cpd}$ and were elevated as hypercyclopean spatial frequency increased or decreased. Vertical gratings required approximately twice as much disparity to be detected as did horizontal gratings. Threshold disparity was minimum for depth reversal intervals exceeding $0.5 \mathrm{sec}$. For shorter intervals, disparity threshold doubled each time interval duration was halved, suggesting temporal integration by the stereoscopic mechanisms up to about 0.5 sec.
\end{abstract}

Stereothresholds, the minimal retinal disparities that permit depth to be perceived, vary with temporal characteristics. They are elevated as the duration of a stimulus exposure is reduced for both local (Langlands, 1929; Ogle \& Weil, 1958) and global stereopsis (Harwerth \& Rawlings, 1977; Uttal, Fitzgerald, \& Eskin, 1975). Stereothresholds increase as the rate of depth reversal increases for continuously viewed global (Beverly \& Regan, 1974a; Regan \& Beverly, 1973a) and local stereoscopic stimuli (Beverly \& Regan, 1974b; Engel, 1970; Richards, 1972; Tyler, 1971) and for pulsed local (Beverly \& Regan, 1974b) and global stereoscopic stimuli (Norcia \& Stevenson, 1982). Suprathreshold depth matches (Regan \& Beverly, 1973b) and the limits of fusion have also been demonstrated to be affected by the temporal characteristics of disparity stimulation (Fender \& Julesz, 1967; Schor \& Tyler, 1981).

In the present experiment, the effect of temporal squarewave modulation of disparity on stereothresholds was examined. To increase the generalizability of these results, certain additional stimulus characteristics were varied systematically-namely, pattern form (gratings vs. checks), pattern orientation (horizontal or vertical), and fundamental hypercyclopean spatial frequency of the "pattern in depth" whose visibility was supported only by global stereopsis.

\section{Observers \\ METHOD}

The observers were three males, aged 27,33 , and 34 years and with acuity correctable to $6 / 6$ or better. The stereoacuity of all three

Supported by NEI Grants EY-03640 to K.D.W. and EY-03781 to J.V.O., by a Biomedical Research Support grant from the University of Florida Division of Sponsored Research to K.D.W., and a Research to Prevent Blindness unrestricted departmental grant to the Department of Ophthalmology of West Virginia University. K. D. White is a member of the Departments of Psychology, Ophthalmology, and Electrical Engineering of the University of Florida. J. V. Odom was in the Department of Ophthalmology. Reprint requests should be directed to him at his present address: Department of Ophthalmology, West Virginia University Medical Center, Morgantown, WV 26506. observers was superior, at least 9" (seconds of arc), as measured on the Bausch and Lomb orthorater. K.W. and V.O. were experienced observers. J.W. was experienced at making auditory, but not visual, psychophysical judgments.

\section{Apparatus}

The patterns in depth (anaglyphic dynamic random element stereograms-ADRES) were generated using a graphics computer (Ohio Scientific Challenger 1P), a custom-built computer interface, and a projection television (Advent 710). The graphics computer generated patterns and controlled the timing between depth reversals. The computer interface generated pseudorandom noise and regulated the relative delay between two noise outputs, thereby determining both the direction of the disparity (crossed, "near," or uncrossed, "far") and its magnitude. Unlike other devices for producing ADRES, in this one the magnitude of disparity was under analog (continuous) control from a maximum of $30^{\prime}$ (arc min) to a minimum of zero. The outputs of the interface controlled the red and green channels of a projection television. See White and Odom (1983) for details of the circuit and its operation. Disassociation of the eyes was maintained by using chromatically selective filters over the eyes. When viewed through these filters, the mean luminances were 51 and $67 \mathrm{~cd} / \mathrm{m}^{2}$ for the red and green element fields, respectively; the interocular luminance ratio was approximately 0.12 $\log$ units.

\section{Procedures}

Observers viewed a screen subtending $18^{\circ} \times 23^{\circ}$ at a distance of $3 \mathrm{~m}$. A frame of element pairs was generated randomly 60 times per second. Each element subtended $2.5^{\prime} \times 3.8^{\prime}$. A no-disparity surround enclosed an $11^{\circ} \times 16^{\circ}$ area in which elements with crossed (C) and uncrossed (U) disparities filled alternate bars of horizontal or vertical gratings or alternate checks of checkerboards. The groups of elements in a pattern of bars or checks appeared nearer (C) or farther (U) than the surround, giving rise to the perception of a pattern in depth. We refer to the patterns in depth and to the spatial frequencies of those patterns as hypercyclopean, because fusion of the constituent cyclopean elements is a precondition to the patterns' visibility (see Tyler, 1975, 1977). The patterns were not visible in any of three control experiments using the chromatic filters: (1) monocular viewing, (2) binocular viewing by stereoblind observers, and (3) binocular viewing by normal observers with their heads tilted to align their eyes vertically.

Reversals in depth were produced by exchanging $C$ for $U$ and $\mathrm{U}$ for $\mathrm{C}$ at intervals ranging from 0.03 to $4.27 \mathrm{sec}(15$ to $0.11 \mathrm{~Hz}$ ). Video synch pulses were counted by the microcomputer to insure fully interlaced frames on the display. Depth reversals were made 
without changes in space-averaged luminance, mean correlation of elements, or any cues other than disparity type (C or $U$ ).

The patterns in depth had mean fundamental hypercyclopean spatial frequencies ranging from 0.55 to 0.13 cycles per degree (cpd). Presentation of stimuli was counterbalanced for pattern form and orientation (horizontal gratings, vertical gratings, or checkerboards), for interval duration within each session, and for spatial frequency ( 0.55 to $0.13 \mathrm{cpd}$ ) across sessions. Form sequence and rate sequence also were partially counterbalanced across sessions. A session that consisted of 24 presentations, each requiring a single threshold judgment, took about $30 \mathrm{~min}$ to complete. Stereothresholds were determined using a method of adjustments. Across sessions, four threshold measurements were taken for each specific stimulus configuration.

The subjects were instructed to accept a no-depth criterion (neither the pattern in depth nor its junction with the surround were visible) in making judgments of depth. Our technique did not permit separate threshold determinations for $\mathrm{C}$ and $\mathrm{U}$ disparities, but the judgment criterion would be rejected if $\mathrm{C}, \mathrm{U}$, or both reached threshold. Threshold measures are reported as the total $(C+U)$ disparity necessary to reject the no-depth criterion.

\section{RESULTS}

The median across sessions of the four stereoacuity threshold settings (logarithm of arc sec) for a particular subject $\times$ pattern type $\times$ orientation $\times$ spatial frequency $x$ interval duration was calculated. The means of the median stereoacuity thresholds, collapsed across pattern type and orientation, are shown for each subject, pattern spatial frequency, and interval between depth reversals in Figure 1. This figure emphasizes the temporal response characteristics of the global stereoscopic mechanism. The results from the three observers are comparable. Stereoacuity threshold decreased as the interval between depth reversals increased, from a maximum when the time between depth reversals was $33 \mathrm{msec}(15 \mathrm{~Hz}$ reversal frequency) to a minimum beyond approximately $500 \mathrm{msec}$ (1 $\mathrm{Hz}$ and lower frequencies).

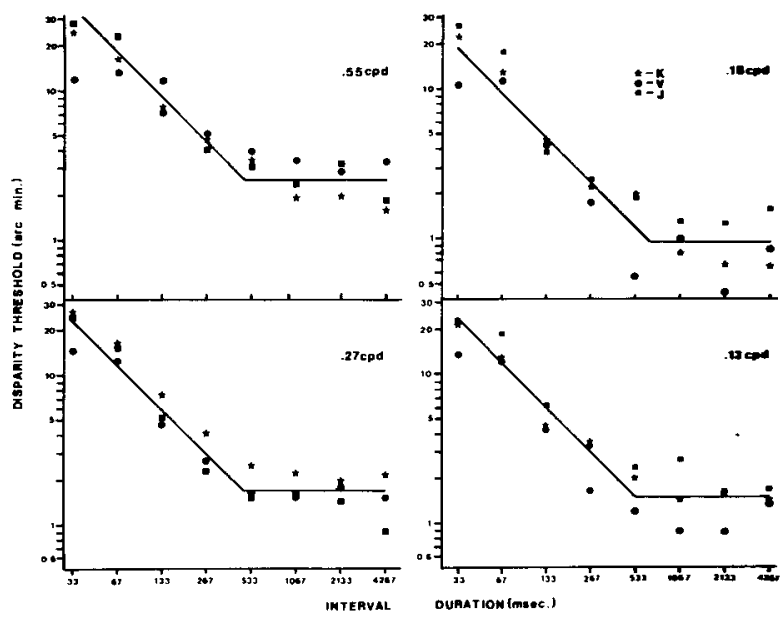

Figure 1. Disparity thresholds as a function of the time between depth reversals. The four panels compare the results for patterns of different fundamental hypercyclopean spatial frequencies, from 0.55 cpd (upper left panel) to 0.13 cpd (lower right panel). Each plotted point represents a mean-median of 12 judgments. Symbol shape identifies data from each observer. Smooth lines depict hypothetical temporal summation up to about 500 msec.

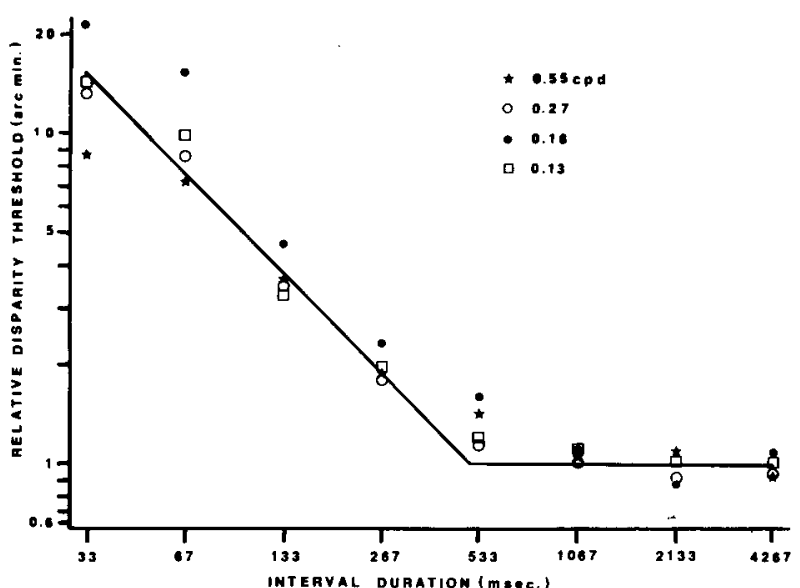

Figure 2. Relative disparity thresholds as a function of the time between depth reversals. Data for the three observers were averages at each spatial frequency $\times$ interval duration, and the averages were normalized to an arbitrary asymptotic value of 1 arc min relative disparity (see the text). Different symbol shapes stand for fundamental hypercyclopean spatial frequencies of the judged patterns. Smooth lines depict expected outcomes from limited temporal integration. Because the duty cycle of the square-wave depth reversals was $50 \%$, time between depth reversals may be transformed to frequency of depth reversals by dividing 1,000 msec by twice the interval in milliseconds. The frequency of depth reversal varies from 0.11 to $15 \mathrm{~Hz}$.

The solid lines in Figure 1 represent perfect temporal integration up to a critical duration. The line segments have slopes of -1 and 0 , respectively. Ordinate heights of the lowest threshold asymptotes (line segments with 0 slope) varied significantly with hypercyclopean spatial frequency, from an intermediate threshold value at .13 cpd $\left(1^{\prime} 15^{\prime \prime}\right)$, to the minimum for the 0.18 -cpd patterns $\left(57.6^{\prime \prime}\right)$ and the maximum for the 0.55-cpd patterns $\left(2^{\prime} 30^{\prime \prime}\right)$, suggesting spatial tuning. The ratio between the maximum and minimum asymptotes was roughly $0.4 \mathrm{log}$ units, or $250 \%$.

The relative disparity thresholds for all the patterns and interval durations used are presented in Figure 2. Data were averaged across subjects, sessions, pattern types, and orientations. The grand mean of median thresholds for interval durations greater than $1.0 \mathrm{sec}$ was calculated for each pattern spatial frequency, so as to estimate the ordinate height of the minimum threshold asymptote. All data for that spatial frequency were then divided by the factor needed to adjust this asymptote to an arbitrary relative value of 1 arc min disparity. Solid lines with slopes of -1 and 0 represent perfect linear temporal integration, assuming a critical duration of approximately $500 \mathrm{msec}$. The good fit between this function and the relative threshold data suggests that a single temporal process may be applicable without regard to the specific spatial frequencies of the stimulus patterns.

A small but statistically reliable $(p<.05)$, anisotropy between vertically oriented and horizontally oriented hypercyclopean gratings was also observed. The ratios of stereothresholds for vertical relative to horizontal grat- 
ings of the same fundamental cyclopean spatial frequency were: K.W., 2.99; V.O., 2.01; and J.W., 1.27. It can be seen that vertical gratings required roughly twice as much disparity to be resolved in depth as did horizontal gratings. Checkerboards having both vertical and horizontal hypercyclopean contours yielded intermediate stereothresholds.

\section{DISCUSSION}

In the present experiment, four findings are noteworthy. First, ADRES stimuli can elicit reliable stereothresholds from selected observers with total disparities $(\mathrm{C}+\mathrm{U})$ in the range of $20^{\prime \prime}$ to $30^{\prime \prime}$. Second, stereothresholds are lowest when judged on hypercyclopean patterns of about 0.18 -cpd fundamental spatial frequency (i.e., spatial tuning of the hypercyclopean stereomechanism). Third, there is a small orientation anisotropy so that vertical patterns are less easily seen in depth than horizontal patterns. Fourth, stereothresholds vary according to the temporal characteristics of disparity modulations.

\section{Stereothreshold Values}

Thresholds for local stereopsis are consistently less than 10 " for normal observers (Westheimer \& McKee, 1980), and with continuous viewing the best observers are reported to have thresholds of 2 " (Berry, 1948; Hirsch, Horowitz, \& Weymouth, 1948; Howard, 1919; Woodburne, 1934). Thresholds for global stereopsis are frequently reported to be greater than $1^{\prime}$ for normal observers (Harwerth \& Rawlings, 1977). In part, the elevated thresholds reported for global stereopsis are an artifactual consequence of the inability to produce disparities less than the element size with most random-element methodologies (Harwerth \& Rawlings, 1977; Westheimer \& McKee, 1980). When this constraint is overcome (as in the present study) and long viewing times are permitted, the reported thresholds for global stereopsis are consistently less than $1^{\prime}$ and frequently on the order of $20^{\prime \prime}$ to 30" (Schumer \& Ganz, 1979; Schumer \& Julesz, 1984; Uttal et al., 1975; this study). Local stereoscopic thresholds for normal observers may be elevated to $20^{\prime \prime}-30$ ", however, as the separation between local elements decreases below $5^{\prime}$ or as pattern elements are reduced in size or defocused slightly (Westheimer \& McKee, 1980). Our ADRES stimuli used such small elements with small separations, and the projection TV was not capable of perfect electronic and optical focus. It seems conceivable, in the light of Westheimer and McKee's (1980) report, that global stereopsis thresholds may approximate those of local stereopsis.

\section{Spatial Frequency Tuning}

We observed the lowest thresholds when patterns with a hypercyclopean spatial frequency of $0.18 \mathrm{cpd}$ were used as stimuli. Both lower and higher spatial frequencies yielded higher thresholds. The presence of a curvilinear relationship between stereothreshold and hypercyclopean spatial frequency suggests spatial tuning. By analogy to experiments using adaptation to luminance gratings or to narrow-band visual noise, several investigators have estimated the bandwidth of hypercyclopean spatial frequency channels (Schumer \& Ganz, 1979; Tyler, 1974, 1975; Tyler \& Julesz, 1978). In general, hypercyclopean channels seem to have a relatively broad passband of about 2 octaves at half-amplitude and seem to be most sensitive to spatial frequencies lower than $1 \mathrm{cpd}$ (Schumer \& Ganz, 1979; Tyler, 1974; this study).

\section{Orientational Anisotropy}

Elevated stereothresholds for vertically versus horizontally oriented hypercyclopean patterns have been reported by Rogers and Graham (1983) and confirmed in the present results. This orientational anisotropy must be interpreted cautiously, though, because a vertical hypercyclopean contour can complicate the horizontal disparity of any element pairs in the dynamically changing stimulus which happen to coincide with the contour. Stimulus artifacts potentially result.

We found, except at the lowest hypercyclopean spatial frequencies, that: (1) vertical gratings showed the highest stereothresholds; (2) the addition of horizontal contours, producing checkerboard patterns, lowered thresholds; and (3) removal of the vertical contours, producing a horizontal grating, lowered stereothresholds even further. This trend of results cannot be explained solely by possible stimulus artifacts at the vertical hypercyclopean contours.

Local and global stereomechanisms are preferentially sensitive to horizontal disparities rather than vertical disparities, and perhaps the horizontal direction retains special significance for hypercyclopean mechanisms as well. However, the present data are too sparse to adequately support such a conjecture, especially with the large individual differences in the orientation anisotropy effect.

\section{Temporal Characteristics of Stereopsis}

Most models of global stereopsis assume that the process is slow because of the relatively complicated neural processing required to generate cyclopean perception (Frisby \& Mayhew, 1980; Julesz \& Chang, 1976; Marr \& Poggio, 1979; Nelson, 1975). Few models attempt to specify the temporal parameters of this process, however.

The effects of exposure duration on stereothresholds have been examined for local and for global stereopsis. Langlands (1929) observed that the threshold for local stereopsis was lowest when the stimuli were presented for durations of $3 \mathrm{sec}$ or longer, that for durations shorter than $100 \mathrm{msec}$ stereothresholds remained at their maximum level (instantaneous threshold). The observation of a linear relationship between stimulus duration and stereothreshold has been replicated (Ogle \& Weil, 1958; Shortess \& Krauskopf, 1961), as has a maximum, instantaneous stereothreshold for exposure durations from 0.2 to $6 \mathrm{msec}$ (Ogle \& Weil, 1958). The general relationship of decreasing stereothreshold with increased viewing duration has also been confirmed for global stereopsis (Har- 
werth \& Rawlings, 1977; Uttal et al., 1975), although an instantaneous threshold for global stereopsis has not been demonstrated (Harwerth \& Rawlings, 1977).

The temporal characteristics of local and global stereopsis have also been examined using reversals in depth. Richards (1951) and Richards (1972) used depth reversals of a single bar, moved symmetrically through the fixation plane. At rates beyond $1 \mathrm{~Hz}$, stereothresholds are considerably elevated (Richards, 1951) and judgments of apparent depth reduced (Richards, 1972); beyond $6 \mathrm{~Hz}$, subjects could no longer make judgments of apparent depth (Richards, 1972). Examination of Richards's figures suggests that their data might be represented by temporal integration functions like those in Figures 1 and 2. Several experiments have examined asymmetric depth reversals of a bar, finding elevated stereothresholds with reduced reversal durations, for durations ranging from 100 to $500 \mathrm{msec}$ ( 5 to $1 \mathrm{~Hz}$ reversal frequency) (Beverly \& Regan, 1974b; Regan \& Beverly, 1973b).

When comparable stimuli are employed, the choice of pulsed or steady state stimulation appears to have minimal effect on estimates of the time (or frequency) at which thresholds become elevated. Thus, use of local line stimuli indicate elevated stereothresholds for pulses less than $100 \mathrm{msec}$ in duration (Beverly \& Regan, 1974a) or for frequencies greater than $5 \mathrm{~Hz}$ if the lines are modulated continuously (Regan \& Beverly, 1973a, 1973b). However, experiments in which global stereoscopic stimuli have been pulsed (Norcia \& Stevenson, 1982) or modulated continuously (Beverly \& Regan, 1974a; this study) have indicated elevated thresholds as duration is decreased below about $500 \mathrm{msec}$ or frequency increased above about $1 \mathrm{~Hz}$. The uniformity of findings for roughly comparable stimulus conditions irrespective of the use of pulses or continuously modulated stimuli suggests that the transient and steady state operation of the stereo mechanisms are comparable, at least near threshold.

The present data are adequately described by assuming that the temporal response characteristics in global stereopsis are those of a simple integrator. By electronic analogy, the temporal characteristics of global stereopsis might be described as a passive, low-pass filter composed of a resistor and a capacitor. Such a "leaky" integrator exhibits the necessary 3-dB per octave attenuation above a characteristic temporal frequency; however, it also shows a gradual loss of sensitivity or roll-off for frequencies just above the passband. Norcia and Stevenson (1982), using disparity pulsed random lines as stimuli for global stereopsis, also have noted a 3-dB per octave attenuation in stereoscopic sensitivity above a characteristic frequency. In their results, as in the present results, there is little evidence of the gradual roll-off expected from a simple RC filter. Therefore, we suggest that a timedomain analysis (linear integration up to a critical duration) provides a more representative description for both sets of findings than a frequency-domain analysis. The bulk of the findings above can be accounted for by such an analysis.
The advantage of a time-domain analysis might be lost if a low temporal frequency loss of sensitivity had been observed. Such results might more easily be described by the frequency-domain analysis of a filter-like mechanism. The absence of stereothreshold elevation at lower temporal frequencies in our experiment cannot be explained solely on the basis of our use of square-wave modulated depth reversals. In experiments in which the mean disparity has been maintained at the plane of fixation, use of sine-wave temporal modulation has not resulted in low temporal frequency stereothreshold elevation (Norcia \& Stevenson, 1982; Regan \& Beverly, 1973b), and direct comparisons of sine- and square-wave stimulation indicate that the two modes of temporal modulation result in similar thresholds (Beverly \& Regan, 1974a, 1974b; Regan \& Beverly, 1973a, 1973b). Stereothreshold elevation has been observed at lower temporal frequencies for both sine- and square-wave modulated stimuli if the mean disparity of the stimulus is outside the plane of fixation (Regan \& Beverly, 1973b; also see Schumer \& Julesz, 1984). However, these results may be confounded by the difficulty in maintaining vergence at the plane of fixation for several seconds when confronted by a powerful stimulus for driving vergence outside of that plane (see Jones \& Stevens, 1982, and Richards, 1972).

Any overly simple model for the stereopsis mechanism must be regarded with caution, since there is evidence for multiple stereo mechanisms. Cells in the visual cortex of monkeys appear to form four classes of disparity detectors: near (C), far (U), excitatory at the plane of fixation, and inhibitory at the plane of fixation (Poggio \& Fischer, 1977). Data on the type and incidence of stereoblindness suggest independent mechanisms for detecting plane of fixation, C, and U disparities (Richards, 1970). Because the present experiment employed repetitive depth reversal as a stimulus, $U$ and $C$ disparities were combined in judgments of stereothresholds. The present results therefore cannot differentiate between a single stereopsis filter and multiple parallel filters, one for each of several stereoscopic channels.

Individual differences were reported by Norcia and Stevenson (1982) in the relationships between disparity thresholds and the fundamental frequency of a partial sinusoid they used to modulate temporal disparity pulses, $\mathrm{U}$ to-C or C-to-U. Those authors conclude that a single temporal response profile may not be representative for all stereomechanisms.

The present results do not show reliable individual differences in response to temporal modulation of the ADRES stimuli. However, in a supplemental experiment, we tested another observer whose stereoacuity was below the superior range of our major observers. We observed: (1) higher asymptote values for the lowest disparity thresholds achieved (about three times larger disparities than the asymptotes in Figure 1); (2) stereothresholds exceeded the asymptotic value only when the interval between depth reversals was reduced to about $130 \mathrm{msec}$ (in 
contrast to roughly $500 \mathrm{msec}$ for the major observers); and (3) results at $130 \mathrm{msec}$ and shorter depth reversal intervals comingled with the data from the major observers. We do not claim that stereo mechanisms of the supplemental observer operate with a compressed time scale, or that this observer's performance limitations tend to obscure relevant data. It seems warranted, however, to voice a suspicion that individual differences may be a prominent factor in the variations of results between studies concerned with temporal characteristics of stereopsis.

\section{Eye Movements and Their \\ Temporal Characteristics}

The fact that the stereoscopic mechanism integrates over time and consequently filters out rapidly occurring and/or rapidly changing visual events has frequently been related to eye movements. Ogle and Weil (1958; see Richards, 1977) noted that the longer the stimulus duration, the more the eyes would move during the interval, because of the natural variability in fixation (Fender \& St. Cyr, 1969; Riggs, Armington, \& Ratliff, 1954; cf. Bishop, 1975, p. 587; Duwaer \& Brink, 1981), and inferred that the stereo mechanism integrates over time, thereby permitting stimulation of more receptors, which would account for the hyperacuity of stereo mechanisms. If stereograms are stabilized on the retinas of the two eyes, both global and local stereopsis continue to support depth perception (Evans \& Clegg, 1967; Fender \& Julesz, 1967; Shortess \& Krauskopf, 1961) and the relationship of stereothresholds to exposure duration remains relatively unaffected. This suggests that eye movements during fixation do not serve to improve stereoacuity. One might regard the variability in fixation as inherent noise obscuring stereoscopic sensations (e.g., Duwaer \& Brink, 1981).

A potentially important correspondence exists between vergence eye movements and the temporal characteristics of stereo mechanisms. Vergence eye movements have a relatively long latency period (approximately $160 \mathrm{msec}$ ) and, unlike saccadic eye movements, may be modified based on information received both during the latency period and during the vergence movement itself (Rashbass $\&$ Westheimer, 1961). Vergence eye movements apparently cease at the same stimulating frequency at which depth reversals become undetectable, suggesting that the vergence system is driven by disparity detectors (Richards, 1972; see also Jones \& Stephens, 1982).

Power spectral density analyses of the eye movements accompanying fixation reveal significant power from $0.2 \mathrm{~Hz}$ or lower up to at least $20 \mathrm{~Hz}$ (R. K. Moore, personal communication, 1981). We also note that fixational eye movements have been reported to have only a low correlation between the eyes (e.g., Fender \& St. Cyr, 1969). We surmise that a significant residual power may be found at frequencies above $2 \mathrm{~Hz}$, even after the power at harmonics of the conjugate eye movements has been parcelled out; this residual is presumably a consequence of vergence instability. Furthermore, we infer that the effects of vergence instability on instantaneous retinal disparities must be filtered out (or averaged out by a limited integration process) so that stereo depth perception is both accurate and stable. Disparity transients might also drive the vergence eye-movement system through feedback. A conceivable consequence of failure to smooth these small disparity transients would be phase-lags and ringing in the feedback loops, with deleterious effects on vergence stability (see Jones \& Stephens, 1982). We also notice that head turns have been reported to transiently disrupt vergence (Steinman \& Collewijn, 1980) but apparently have little effect upon 3-D space constancy (see Ogle, 1962).

\section{SUMMARY}

Using a new stimulus, square-wave reversal of squarewave hypercyclopean gratings created by means of ADRES, the relation between thresholds for global stereopsis and the interval between depth reversals were explored. The findings are well described by a timelimited linear integration, similar in form to Bloch's law, although the critical duration is about five times greater. Temporal integration in global stereopsis would attenuate the effects of rapidly occurring or rapidly changing visual events, and might be related to characteristics of fixation instability and vergence eye-movement control.

In the present study, it was also observed that global stereoscopic thresholds may be as low as $20^{\prime \prime}$ to $30^{\prime \prime}$. The global stereo system is most sensitive to hypercyclopean spatial frequencies below $0.5 \mathrm{cpd}$ and to horizontally oriented hypercyclopean contours.

\section{REFERENCES}

BERRY, R. M. (1948). Quantitative relations among vernier, real depth, and stereoscopic depth acuities. Journal of Experimental Psychology, 38, 708-721.

BeverLy, K. I., Regan, D. (1974a). Temporal integration of disparity information in stereoscopic perception. Experimental Brain Research, 19, 228-232.

BeVerLy, K. I., \& RegaN, D. (1974b). Visual sensitivity to disparity pulses: Evidence for directional selectivity. Vision Research, 14, 357-361.

Bishop, P. O. (1975). Binocular vision. In R. A. Moses (Ed.), Adler's physiology of the eye (pp. 558-614). St. Louis: Mosby.

DUWAER, A. L., \& BRINK, G. VAN DEN (1981). Foveal diplopia thresholds and fixation disparities. Perception \& Psychophysics, 30, 321-329.

ENGEL, G. R. (1970). An investigation to brief stereoscopic stimuli. Quarterly Joumal of Experimental Psychology, 22, 148-166.

Evans, C. R., CleGG, J. M. (1967). Binocular depth perception of Julesz patterns viewed as perfectly stabilized retinal images. Nature, 215, 893-895.

Fender, D. K., \& Julesz, B. (1967). Extension of Panum's fusional area in binocularly stabilized vision. Joumal of the Optical Society of America, 57, 819-830.

FENDER, D. K., \& ST. CYR, G. J. (1969). The interplay of drifts and flicks in binocular fixation. Vision Research, 9, 245-265.

Frisby, J. P., MaYhew, J. E. W. (1980). Spatial frequency tuned channels: Implications for structure and function from psychophysical and computational studies of stereopsis. Philosophical Transactions of the Royal Society, London, Series B, 290, 95-116. 
HARWERTh, R. S., RAWLings, S. C. (1977). Viewing time and stereoscopic threshold with random-dot stereograms. American Journal of Optometry and Physiological Optics, 54, 452-457.

Hirsch, M. J., Horowitz, M., \& WeYMOUTH, F. W. (1948). Distance discrimination: III. Effect of rod width on threshold. Archives of Ophthalmology, 39, 325-332.

HowARD, H. J. (1919). A test for the judgement of distance. American Journal of Ophthalmology, 2, 656-675.

Jones, R., \& STEPHENS, G. (1982). Control of disparity-vergence eye movement. Investigative Ophthalmology and Visual Science, 22, (Suppl.), 84P.

Julesz, B., \& ChANG, J. J. (1976). Interaction between pools of binocular disparity detectors tuned to different disparities. Biological Cybernetics, 22, 107-119.

LANGLANDS, N. M. S. (1929). Reports of the committee upon physiology of vision. IV. Experiment on binocular vision. London: H. M. Stationery Office.

MARR, D., \& PoGgio, T. (1979). A computational theory of human stereo vision. Proceedings of the Royal Society $(B), 204,301-328$.

NeLson, J. I. (1975). Globality and stereoscopic fusion in binocular vision. Journal of Theoretical Biology, 49, 1-88.

Norcin, A. M., \& Stevenson, S. B. (1982). Temporal modulation transfer functions for changing disparities in global stereopsis. Investigative Ophthalmology and Visual Science, 22 (Suppl.), 125P.

OGLE, K. N. (1962). Spatial localization through binocular vision. In H. Davson (Ed.), The eye: Vol. 4. Visual optics and the optical space sense (pp. 271-324). New York: Academic Press.

OGLE, K. N., WEIL, M. P. (1958). Stereoscopic vision and the duration of the stimulus. Archives of Ophthalmology, 59, 4-17.

Poggio, G. F., \& Fischer, B. (1977). Binocular interaction and depth sensitivity in striate and prestriate cortex of behaving rhesus monkey. Journal of Neurophysiology, 40, 1392-1405.

Rashbass, C., \& Westheimer, G. H. (1961). Disjunctive eye movements. Journal of Physiology, 159, 149-170.

REgAN, D., BeverLY, K. I. (1973a). The dissociation of sideways movements from movements in depth: Psychophysics. Vision Research, 13, 2403-2415.

Regan, D., \& BeVERLy, K. I. (1973b). Some dynamic features of depth perception. Vision Research, 13, 2369-2379.

RICHARDS, W. J. (1951). The effect of alternating views of the test object on vernier and stereoscopic thresholds. Journal of Experimental Psychology, 42, 376-383.

RICHARDS, W. (1970). Stereopsis and stereoblindness. Experimental Brain Research, 10, 380-388.

RICHARDS, W. (1972). Response functions for sine- and square-wave modulations of disparity. Journal of the Optical Society of America, 62, $907-911$.
RICHARDS, W. (1977). Stereopsis with and without monocular contours. Vision Research, 17, 967-969.

Riggs, L. A., Armington, J. C., \& RatlifF, F. (1954). Motions of the retinal image during fixation. Journal of the Optical Society of America, 44, 315-321.

Rogers, B. J., Graham, M. E. (1983). Anisotropies in the perception of three-dimensional surfaces. Science, 21, 1409-1411.

SCHOR, C. M., \& TYLER, C. W. (1981). Spatio-temporal properties of Panum's fusional area. Vision Research, 21, 683-692.

SChUMER, R., \& GANZ, L. (1979). Independent stereoscopic channels for different extents of spatial pooling. Vision Research, 19, 1303-1314.

SChUMeR, R. A., Julesz, B. (1984). Binocular disparity modulation sensitivity to disparities offset from the plane of fixation. Vision Research, 24, 533-542.

Shortess, G. K., \& KrausKopf, J. (1961). Role of involuntary eye movements in stereoscopic acuity. Journal of the Optical Society of America, 51, 555-559.

Steinman, R. M., \& Collewijn, H. (1980). Binocular retinal image motion during active head rotation. Vision Research, 20, 415-430.

TYLER, C. W. (1971). Stereoscopic depth movement: Two eyes less sensitive than one. Science, 174, 958-961.

TYLER, C. W. (1974). Depth perception in disparity gratings. Nature, 251, 140-142.

Tyler, C. W. (1975). Stereoscopic tilt and size aftereffects. Perception, 4, 187-192.

TYLER, C. W. (1977). Spatial limitations of human stereoscopic vision. Proceedings of the Society of Photo-Optical Instrumentation Engineers, 120, 36-42.

TYLER, C. W., \& JULESZ, B. (1978). Binocular cross-correlation in time and space. Vision Research, 18, 101-105.

Uttal, W. R., Fitzgerald, J., \& Eskin, T. E. (1975). Parameters of tachistoscopic stereopsis. Vision Research, 16, 705-712.

Westheimer, G., \& MCKeE, S. P. (1980). Stereogram design for testing local stereopsis. Investigative Ophthalmology and Vision Science, 19, 802-809.

WhITE, K. D., \& ODOM, J. V. (1983). Dynamic random-element stereograms: A microprocessor application. Documenta Ophthalmologica Proceedings Series, 37, 241-246.

WoODBURNe, L. S. (1934). The effect of a constant visual angle upon the binocular discrimination of depth differences. American Journal of Psychology, 46, 273-286.

(Manuscript received September 24, 1984; revision accepted for publication December 31, 1984.) 